\title{
Systematic Construction of Real Lapped Tight Frame Transforms
}

\author{
Aliaksei Sandryhaila, Student Member, IEEE, Amina Chebira, Member, IEEE, Christina Milo, \\ Jelena Kovačević, Fellow, IEEE, and Markus Püschel, Senior Member, IEEE
}

\begin{abstract}
We present a constructive algorithm for the design of real lapped equal-norm tight frame transforms. These transforms can be efficiently implemented through filter banks and have recently been proposed as a redundant counterpart to lapped orthogonal transforms, as well as an infinite-dimensional counterpart to harmonic tight frames. The proposed construction consists of two parts: First, we design a large class of new real lapped orthogonal transforms derived from submatrices of the discrete Fourier transform. Then, we seed these to obtain real lapped tight frame transforms corresponding to tight, equal-norm frames. We identify those frames that are maximally robust to erasures, and show that our construction leads to a large class of new lapped orthogonal transforms as well as new lapped tight frame transforms.
\end{abstract}

Index Terms-Bases, DFT, filter banks, frames, lapped orthogonal transforms, orthonormal, paraunitary matrices, tight.

\section{INTRODUCTION}

$\mathbf{O}$ VER the past decades, redundancy has become a common tool in signal processing and communications and found its way into signal representations through frames [1]-[3]. Nowadays, frames serve a wide range of applications from robust transmission to denoising (see [4] and references therein) to the classification of diverse biomedical image datasets [5]-[7]. Motivated by the need of having frame families dedicated to a spectrum of applications not considered before, we seek to design new classes of frames.

We consider a frame to be a redundant set of vectors $\left\{\varphi_{i}\right\}$, $i \in \mathbb{Z}$, which span $\ell^{2}(\mathbb{Z})$. A signal $x \in \ell^{2}(\mathbb{Z})$ is expanded into the frame using a transform, which computes the signal projection coefficients. The original signal is then reconstructed using the corresponding inverse transform

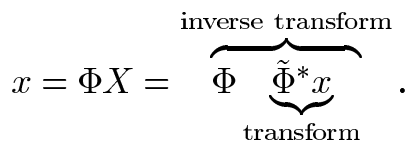

Manuscript received July 28, 2009; accepted December 18, 2009. Date of publication January 29, 2010; date of current version April 14, 2010. The associate editor coordinating the review of this manuscript and approving it for publication was Prof. Xiang-Gen Xia. This work was supported in part by NSF Grants CCF-0634967 and CCF-0515152.

A. Sandryhaila, C. Milo, and M. Püschel are with the Department of Electrical and Computer Engineering, Carnegie Mellon University, Pittsburgh, PA 15213-3890 USA (e-mail: asandryh@andrew.cmu.edu; cmilo@andrew.cmu.edu; pueschel@ece.cmu.edu).

A. Chebira is with the School of Computer and Communications Science, Ecole Polytechnique Fédérale de Lausanne, Lausanne 1015, Switzerland (e-mail: amina.chebira@epfl.ch).

J. Kovačević is with the Departments of Electrical and Computer Engineering and Biomedical Engineering, Carnegie Mellon University, Pittsburgh, PA 15213-3890 USA (e-mail: jelenak@cmu.edu).

Color versions of one or more of the figures in this paper are available online at http://ieeexplore.ieee.org.

Digital Object Identifier 10.1109/TSP.2010.2041865
Here $(\cdot)^{*}$ is the Hermitian transpose. Both $\Phi$ and its $d u a l \tilde{\Phi}$ can be seen as infinite matrices, a view that we take in our construction. The frame vectors $\varphi_{i}$ are the columns of $\Phi$.

Which frame properties should we look for? Our design criteria lead us to search for what we call lapped tight frame transforms (LTFTs). These frames should be as follows.

- Computationally efficient: They can be implemented with filter banks. As an additional benefit, they have real coefficients.

- Tight: $\tilde{\Phi}=\Phi$, so that the signal reconstruction is trivial, since $\Phi \Phi^{*}=I$.

- Equal norm: $\left\|\varphi_{i}\right\|=\left\|\varphi_{j}\right\|$ for any $i, j \in \mathbb{Z}$.

- Lapped: The support of each $\varphi_{i}$ is longer than a single block of the signal processed by the filter bank.

- Maximally robust to erasures (when possible): A signal can be reconstructed after a partial data loss. We provide a formal definition later.

The above requirements resemble those of the nonredundant LTFT counterparts that inspired this work: lapped orthogonal transforms (LOTs) [8]. LOTs are expansions into orthonormal bases (counterpart of tight frames) and computationally efficient since they can be implemented with filter banks. They have basis vectors of overlapping support to eliminate blocking artifacts. In our previous work [9], we constructed LTFTs from LOTs by a process called seeding, a special form of submatrix extraction.

When constructing LTFTs from known LOTs in [9], we noticed that these LOTs have similar structure, which we exploit here to systematically construct a large class of real LOTs from specific submatrices of discrete Fourier transform (DFT) matrices. We then use seeding to obtain real LTFTs from LOTs. We prove that the corresponding frames are equal-norm, tight, and that many of them are maximally robust to erasures. We estimate the total number of the constructed LOTs and LTFTs, and provide examples to illustrate our method. In addition, we demonstrate that some of the known real LOTs can be constructed using our algorithm. Our systematic method is flexible, leads to a large number of previously unknown LOTs and LTFTs, and implicitly ensures the desirable properties we listed above.

Related work includes [10], where the authors propose a transform derived from the extended lapped complex transform [11]. They use a change of parameters to derive the decomposition vectors from the extended lapped complex transform, ensure that the decomposition is invertible, and describe the construction of the inverse. While in spirit this approach is similar to ours, it does not use seeding and leads to a completely different LTFT (the corresponding inverse filter 


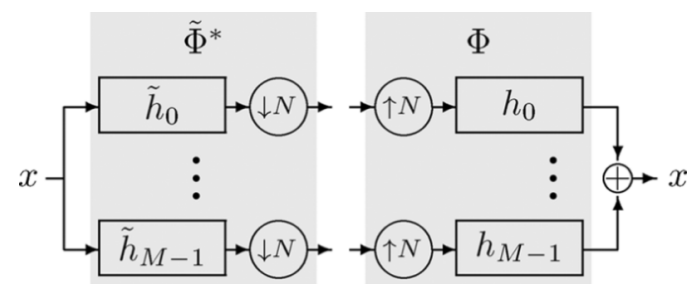

Fig. 1. An $M$-channel filter bank sampled by $N$ implementing a basis ( $M=$ $N)$ or a frame $(M>N)$ expansion. The analysis part computes the transform $X=\tilde{\Phi}^{*} x$; and the synthesis part computes the inverse transform $x=\Phi X$.

bank is optimized to process seismic data [12]). The same authors have also developed a 2-D nonseparable LTFT [13].

Another well-known family of tight frames, the tight Weyl-Heisenberg frames [14], can also be viewed as a special class of LTFTs with arbitrary overlap. These frames are constructed from a prototype window function by translating it in time and modulating in frequency. They can also be implemented with oversampled multichannel filter banks, in which all filters are modulates of one prototype filter. To demonstrate this, we identify a subclass of LTFTs constructed in this paper that are tight Weyl-Heisenberg frames.

\section{BACKGROUND}

In this section, we discuss signal transforms that can be implemented with multichannel filter banks. Such transforms can be interpreted as expansions into bases or frames, implemented with critically sampled or oversampled filter banks, respectively. Our focus is on basis and frame vectors with overlapping support to avoid blocking effects. Finally, we describe the seeding process and discuss the construction of tight frames by seeding basis matrices.

\section{A. Filter Banks}

Consider an $M$-channel filter bank, shown in Fig. 1. Each channel consists of an analysis filter $\tilde{h}_{m}$ and synthesis filter $h_{m}(m=0, \ldots, M-1)$, and down- and upsamplers by $N$. If $M=N$, the filter bank is called critically sampled; if $M>N$, it is oversampled. We assume all analysis and synthesis filters $\tilde{h}_{m}=\left(\tilde{h}_{m, 0}, \ldots, \tilde{h}_{m, L-1}\right)$ and $h_{m}=\left(h_{m, 0}, \ldots, h_{m, L-1}\right)$ have the same length $L=q N$ for some $q \in \mathbb{N}$ (this requirement is not restrictive as long as all filters have finite support). For a signal $x$, the operation of the filter bank can be described via matrix-vector products as shown in (1): the transform $X=\tilde{\Phi}^{*} x$ is filtering followed by downsampling and the inverse transform $x=\Phi X$ is upsampling followed by filtering. $\Phi$ has the form

$$
\Phi=\left(\begin{array}{ccccccc}
\ddots & \vdots & \vdots & \vdots & \vdots & \vdots & \ddots \\
\ldots & \Phi_{0} & 0 & \ldots & 0 & 0 & \ldots \\
\ldots & \Phi_{1} & \Phi_{0} & \ldots & 0 & 0 & \ldots \\
\ldots & \vdots & \vdots & \vdots & \vdots & \vdots & \ldots \\
\ldots & \Phi_{q-1} & \Phi_{q-2} & \ldots & \Phi_{0} & 0 & \ldots \\
\ldots & 0 & \Phi_{q-1} & \ldots & \Phi_{1} & \Phi_{0} & \ldots \\
\ddots & \vdots & \vdots & \vdots & \vdots & \vdots & \ddots
\end{array}\right)
$$

where each block $\Phi_{r}, 0 \leq r \leq q-1$, is the $N \times M$ matrix

$$
\Phi_{r}=\left(\begin{array}{ccc}
h_{0, r N} & \ldots & h_{M-1, r N} \\
\vdots & \ddots & \vdots \\
h_{0, r N+N-1} & \ldots & h_{M-1, r N+N-1}
\end{array}\right)
$$

Note that the synthesis filters form the columns of $\Phi$.

For (1) to hold, we must have $\Phi \tilde{\Phi}^{*}=I$. In this paper, we only consider self-dual bases and frames, meaning $\Phi=\tilde{\Phi}$ or

$$
\Phi \Phi^{*}=I .
$$

We can rewrite (4) in the $z$-domain using polyphase analysis. Namely, we define the $N \times M$ polyphase matrix $\Phi_{p}(z)$ as ${ }^{1}$

$$
\Phi_{p}(z)=\sum_{r=0}^{q-1} \Phi_{r} z^{-r}
$$

with $\Phi_{r}$ as defined in (3). We say $\Phi_{p}(z)$ has degree $q-1$, since any polynomial in $\Phi_{p}(z)$ has degree at most $q-1$. Using (5), (4) is equivalent to $\Phi_{p}(z)$ being paraunitary:

$$
\Phi_{p}(z) \Phi_{p}^{*}(z)=I
$$

Here, $\Phi_{p}^{*}(z)$ represents the Hermitian transpose of a polyphase matrix of $\Phi(z)$, in which coefficients are complex-conjugated, $z^{-1}$ is replaced by $z$, and the matrix is transposed. A paraunitary square matrix is unitary on the unit circle.

If we consider the columns of $\Phi$ as vectors in $\ell^{2}(\mathbb{Z})$, then (4) requires these vectors to form either an orthonormal basis (for $M=N$ ) or a tight, self-dual frame (for $M>N$ ) in $\ell^{2}(\mathbb{Z})$. We will often emphasize the special case of a basis by denoting $\Phi$ with $\Psi$. Correspondingly, the base vectors are denoted with $\varphi$ for frames or $\psi$ for bases.

In summary, oversampled filter banks correspond to frames in $\ell^{2}(\mathbb{Z})$, whose elements form the columns of $\Phi$ in (2). The converse is also true. This class of frames is called filter bank frames.

We have three equivalent representations of filter bank frames, and, by slight abuse of notation, we will use them interchangeably as convenient and refer to all of them as frames:

1) a set of vectors $\left\{\varphi_{i}\right\}_{i \in \mathbb{Z}}$ spanning $\ell^{2}(\mathbb{Z})$;

2) an infinite matrix $\Phi$ as in (2);

3) a polyphase matrix $\Phi_{p}(z)$ as in (5).

We will also encounter finite frames, that is, spanning sets of $\mathbb{C}^{N}$ or $\mathbb{R}^{N}$, and will view them equivalently as $N \times M$ matrices, $M \geq N$. A finite basis hence corresponds to a square matrix.

For a given frame $\Phi, X=\Phi^{*} x$ is the associated transform that computes the vector of projection coefficients with respect to $\Phi$, as shown in (1). Depending on the value of $q, \Phi$ processes the signal $x$ either in nonoverlapping $(q=1)$ or overlapping $(q \geq 2)$ blocks, thus leading to either blocked or lapped transforms $\Phi^{*}$. These cases are visualized in Fig. 2 and discussed next.

\footnotetext{
${ }^{1}$ The subscript $p$ will always denote a polyphase matrix in this paper and should not be confused with subscripts denoting submatrices as in (2).
} 


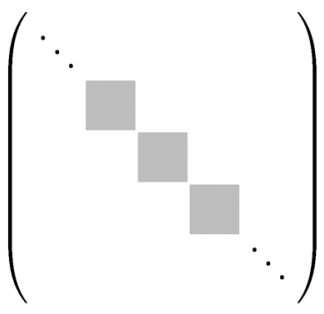

(a)

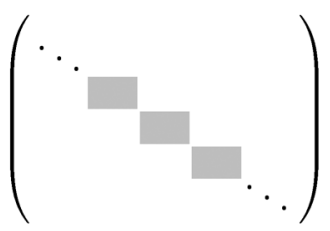

(c)

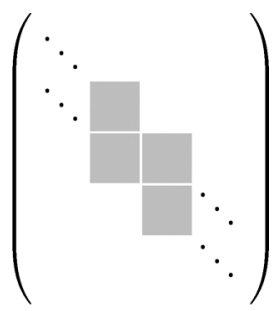

(b)

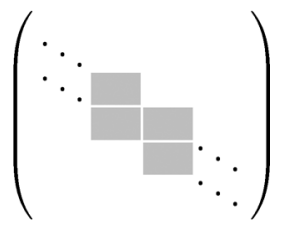

(d)
Fig. 2. The infinite matrix $\Phi$ in (2) in four different scenarios. The columns of $\Phi$ are the basis/frame vectors. In this paper we assume $\Phi \Phi^{*}=I ; \Phi^{*}$ is the transform that computes the corresponding coefficients of a signal $x$. (a) $\Phi=\Psi$ for basis expansion with block transform, (b) $\Phi=\Psi$ for basis expansion with lapped transform $(q=2)$, (c) $\Phi$ for frame expansion with block transform, (d) $\Phi$ for frame expansion with lapped transform $(q=2)$.

\section{B. Basis Expansions $(\Phi=\Psi)$}

Basis Expansions With Blocked Transforms: In a criticallysampled filter bank $(M=N)$ with filters of length equal to the sampling factor $L=N=M(q=1)$,

$$
\Psi=\operatorname{diag}\left(\ldots, \Psi_{0}, \Psi_{0}, \ldots\right)
$$

is a block-diagonal matrix with copies of $\Psi_{0}$ on the diagonal, as visualized in Fig. 2(a). In this case, (4) is equivalent to $\Psi_{0} \Psi_{0}^{*}=$ $I_{M}$, that is, $\Psi_{0}$ is an orthonormal basis in $\mathbb{C}^{M}$. The filter bank processes an infinite signal $x \in \ell^{2}(\mathbb{Z})$ by applying $\Psi_{0}$ to successive nonoverlapping blocks of $M$ signal elements. Since signal blocks are processed as independent signals, and the results are then concatenated, blocking effects occur due to boundary discontinuities. A well-known example of a blocked transform uses $\Psi_{0}^{*}=\mathrm{DFT}_{M}$; others include the use of discrete cosine and sine transforms or the discrete Hartley transform.

In the case of the DFT,

$$
\Psi_{0}^{*}=\operatorname{DFT}_{M}=\frac{1}{\sqrt{M}}\left[\omega_{M}^{m k}\right]_{0 \leq m, k<M}, \quad \omega_{M}=e^{-2 \pi j / M}
$$

Basis Expansions With Lapped Transforms: To avoid blocking artifacts, basis vectors with longer support can be used, as is the case with LOTs. They can be viewed as a class of $M$-channel critically-sampled filter banks, originally developed for filters of length $L=2 N=2 M$ and later generalized to arbitrary integer multiples of $N$ [8].

In this paper, we focus on LOTs $\Psi^{*}$ with basis vector support $L=2 N=2 M(q=2)$ whose bases $\Psi$ are visualized in Fig. 2(b). The only nonzero blocks in (2) are $\Psi_{0}$ and $\Psi_{1}$; hence, (5) yields a polyphase matrix of degree $q-1=1$ :

$$
\Psi_{p}(z)=\Psi_{0}+z^{-1} \Psi_{1}
$$

Since $\Psi_{p}(z)$ is square, (6) is equivalent to

$$
\begin{aligned}
& \Psi_{0} \Psi_{0}^{*}+\Psi_{1} \Psi_{1}^{*}=I \\
& \Psi_{0} \Psi_{1}^{*}=\Psi_{1} \Psi_{0}^{*}=0 .
\end{aligned}
$$

We use these conditions later to show that the new transforms we construct are indeed LOTs.

Two main classes of LOTs exist that use either complex exponentials or cosines in their basis vectors. They are used in a variety of applications. For example, the Malvar LOT [15] is well-suited for noise suppression and echo cancellation, while the Young-Kingsbury LOT [11] was introduced for motion estimation applications. Below we list four known families of LOTs $\Psi^{*}$ by specifying a block row of this matrix, that is, the $M \times 2 M$ matrix $\left[\Psi_{0}^{*} \Psi_{1}^{*}\right]$. The index range in each case is $0 \leq m<M, 0 \leq k<2 M$. A block column of the corresponding basis $\Psi$ is obtained by Hermitian conjugation.

- Princen-Johnson-Bradley LOT [16]:

$$
\frac{1}{\sqrt{M}}\left[\cos \left(\frac{\pi(2 m+1)(2 k-M+1)}{4 M}\right)\right]
$$

- Oddly-Modulated DCT LOT:

$$
\frac{1}{\sqrt{M}}\left[\cos \left(\frac{\pi(2 m+1)(2 k+M+1)}{4 M}\right)\right]
$$

- Young-Kingsbury LOT [11]:

$$
\frac{1}{\sqrt{M}}\left[(-1)^{m} \sin \left(\frac{(2 k+1) \pi}{4 M}\right) j \omega_{8 M}^{(2 m+1)(2 k+1)}\right]
$$

- Malvar Complex LOT [15]:

$$
-\sqrt{\frac{2}{M}}\left[\sin \left(\frac{(2 k+1) \pi}{4 M}\right) \omega_{8 M}^{-(2 m+1)(2 k+1+M)}\right] .
$$

\section{Frame Expansions}

In the previous section, we explained how critically-sampled filter banks compute basis expansions. Similarly, oversampled filter banks compute frame expansions.

For frames, the property (4), $\Phi \Phi^{*}=I$, is called tightness [17]. ${ }^{2}$ Tight frames can be constructed from orthonormal bases using the Naimark theorem [18], [19].

Theorem 1: A set $\left\{\varphi_{i}\right\}_{i \in \mathcal{I}}$ is a tight frame for a Hilbert space $\mathbb{H}$ if and only if there exists another Hilbert space $\mathbb{K} \supset \mathbb{W}$ with an orthonormal basis $\left\{\psi_{i}\right\}_{i \in \mathcal{I}}$, so that the orthogonal projection $P$ of $\mathbb{K}$ onto $\mathbb{H}$ satisfies: $P \psi_{i}=\varphi_{i}$, for all $i \in \mathcal{I}$.

One example of an orthogonal projection is the canonical projection that simply omits coordinates and is called seeding [20].

In the finite case, seeding yields a frame $(N \times M$ matrix $) \Phi$ for $\mathbb{C}^{N}$ by omitting rows from a basis ( $M \times M$ matrix) $\Psi$ of $\mathbb{C}^{M}$. Conversely, every finite frame can be obtained this way. ${ }^{3}$

To seed in the infinite case considered here, we extend this approach to polyphase matrices $\Psi_{p}(z)$.

${ }^{2}$ Note that in general, a tight frame is also one for which $\Phi \Phi^{*}=c I$; however, since $c$ can be pulled into $\Phi$, we consider only $c=1$ here

${ }^{3}$ Just extend $\Phi$ with rows to an invertible square matrix. 
Definition 1: A frame $\Phi_{p}(z)$ is obtained by seeding from a basis $\Psi_{p}(z)$, if it is constructed from $\Psi_{p}(z)$ by preserving only a subset of the rows of $\Psi_{p}(z)$. This is written as $\Phi_{p}(z)=$ $\Psi_{p}(z)[\mathcal{I}]$, where $\mathcal{I}$ is the set of indexes of the retained rows.

In particular, for $q=1$, seeding constructs frames of the form in Fig. 2(c) from bases of the form in Fig. 2(a). Conversely, every such frame can be constructed this way.

For $q>1$, seeding constructs frames of the form in Fig. 2(d) from bases of the form in Fig. 2(b) (the example in the figure is for $q=2$ ). However, in this case, it is unclear whether the converse is true.

The following result is a special case of Theorem 1.

Lemma 1: Seeding an orthonormal basis (paraunitary) $\Psi_{p}(z)$ yields a tight frame $\Phi_{p}(z)$.

Next, we discuss the blocked and lapped frame expansions in Fig. 2(c) and (d) in greater detail.

Frame Expansions With Blocked Transforms: If $q=1$, then, as visualized in Fig. 2(c),

$$
\Phi=\operatorname{diag}\left(\ldots, \Phi_{0}, \Phi_{0}, \ldots\right) \text {. }
$$

The difference from (7) is that $\Phi_{0}$ is now rectangular: $\Phi_{0} \in$ $\mathbb{C}^{N \times M}$, and can be viewed as an $M$-element frame in $\mathbb{C}^{N}$. Hence, if it is tight, it can be constructed from an orthogonal basis in $\mathbb{C}^{M}$ by seeding.

As an example, harmonic tight frames (HTFs) are obtained by preserving the first $N$ rows of $\Psi_{0}=\mathrm{DFT}_{M}^{*}$, that is, $\Phi_{0}=$ $\mathrm{DFT}_{M}^{*}[0, \ldots, N-1]$. $\Phi_{0}$ is a frame for $\mathbb{C}^{N}$; the corresponding $\Phi$ in (14) is a frame for the complex $\ell^{2}(\mathbb{Z})$.

Similarly, real HTFs can be obtained by seeding from the real DFT (RDFT) [20]. For example, the Mercedes-Benz frame

$$
\Phi_{0}=\left(\begin{array}{ccc}
0 & -1 / \sqrt{2} & 1 / \sqrt{2} \\
\sqrt{2 / 3} & -1 / \sqrt{6} & -1 / \sqrt{6}
\end{array}\right)
$$

can be seeded from the orthogonal

$$
\operatorname{RDFT}_{3}=\left(\begin{array}{crr}
1 / \sqrt{3} & 1 / \sqrt{3} & 1 / \sqrt{3} \\
\sqrt{2 / 3} & -1 / \sqrt{6} & -1 / \sqrt{6} \\
0 & -1 / \sqrt{2} & 1 / \sqrt{2}
\end{array}\right)
$$

by retaining (and exchanging) the last two rows.

Frame Expansions With Lapped Transforms: Projecting signals onto frame vectors with nonoverlapping support leads to similar blocking artifacts as for orthonormal bases. We thus use the same approach as for orthonormal bases in Section II-B and consider frames in $\ell^{2}(\mathbb{Z})$ with vector support $L=2 N$, visualized in Fig. 2(d).

As in (8), the resulting polyphase matrix $\Phi_{p}(z)$ has degree 1:

$$
\Phi_{p}(z)=\Phi_{0}+z^{-1} \Phi_{1},
$$

and the tightness condition $\Phi \Phi^{*}=I$ is equivalent to $\Phi_{p}(z)$ being paraunitary (6).

In [9], we constructed LTFTs by seeding the polyphase matrix $\Psi_{p}(z)$ of an LOT basis:

$$
\Phi_{p}(z)=\Psi_{p}(z)[\mathcal{I}]
$$

By the Naimark theorem, the constructed frames are tight; this is why we named them lapped tight frame transforms. We will follow later the same procedure here to derive LTFTs from LOT bases. First, we introduce the frame properties we consider.

\section{Frame Properties}

Apart from tightness, other frame properties are often desirable, such as [4] the following.

- Equal norm: These are frames with basis vectors of the same norm, $\left\|\varphi_{i}\right\|=\left\|\varphi_{j}\right\|$, for $i, j \in \mathcal{I}$. Since in the real world, the squared norm of a vector is usually associated with its energy, equal norm is required in situations where equal-energy signals are desirable.

- Maximal robustness: An $N \times M$ frame $\Phi_{p}(z)$ is maximally robust to erasures, if and only if any $N \times N$ submatrix of $\Phi_{p}(z)$ has the full rank on the unit circle. This requirement arose in using frames for robust transmission [21], where the loss of up to $M-N$ transform coefficients over the transmission channel would not prevent the complete reconstruction of the original signal. The loss of coefficients translates into removal of the corresponding set of $M-N$ columns in $\Phi_{p}(z)$ and the ability to reconstruct translates into the remaining matrix being invertible.

We can construct new frames from old ones by appropriate transformations that preserve the desired properties. Below we list such transformations in the polyphase domain (polynomial counterpart of the discussion for scalar matrices [20]).

Proposition 1: Assume all the matrix products below are compatible and $\Phi_{p}(z)$ is a frame. Then,

i) $U_{p}(z) \Phi_{p}(z) V_{p}(z)$ is a frame, for any $U_{p}(z), V_{p}(z)$ of full rank on the unit circle;

ii) if $\Phi_{p}(z)$ is a tight (unit-norm tight) frame, then $a U_{p}(z) \Phi_{p}(z) V_{p}(z)\left(U_{p}(z) \Phi_{p}(z) V_{p}(z)\right)$ is also a tight (unit-norm tight) frame, for any paraunitary matrices $U_{p}(z), V_{p}(z)$ and $a \neq 0$;

iii) if $\Phi_{p}(z)$ is a maximally robust frame, then $U_{p}(z) \Phi_{p}(z) D_{p}(z)$ is also a maximally robust frame, for any diagonal matrix $D_{p}(z)$ and any matrix $U_{p}(z)$, both of full rank on the unit circle.

\section{CONSTRUCtion OF NEW LOTS AND LTFTS}

Our goal is to design real filter bank frames that are tight, equal-norm, and maximally robust to erasures. We do this by starting from a polyphase matrix closely related to the DFT. We first show that particular submatrices of this matrix yield new LOTs $\Psi$ (bases); we then use seeding to obtain the desired frames $\Phi$ and hence LTFTs $\Phi^{*}$.

\section{A. Construction of New Real LOTs}

In Section II-B, we showed that a real LOT basis corresponds to a real square paraunitary polyphase matrix $\Psi_{p}(z)$ of degree $q-1$. Although in general $\Psi_{p}(z)$ is paraunitary if and only if it is unitary on the entire unit circle $|z|=1$, for a real $\Psi_{p}(z)$ of degree $q-1=1$, it suffices to check only two conditions.

Lemma 2: Let $\Psi_{p}(z)$ be a real $M \times M$ polyphase matrix of degree 1, that is, $\Psi_{p}(z)=\Psi_{0}+z^{-1} \Psi_{1}$, where $\Psi_{0}, \Psi_{1} \in$ $\mathbb{R}^{M \times M}$. Then, $\Psi_{p}(z)$ is paraunitary if and only if $\Psi_{p}(1)$ and $\Psi_{p}(j)$ are unitary. 
$\operatorname{DFT}_{p, K}(z) \stackrel{\text { submatrix }}{\longrightarrow} \Psi_{p}(z) \stackrel{\text { seed }}{\longrightarrow} \Phi_{p}(z)$

Fig. 3. Construction of frames for $q=2$.

Proof: " $\Rightarrow$ " is immediate. To prove " $\Leftarrow$ ", let $\Psi_{p}(1)=$ $\Psi_{0}+\Psi_{1}$ and $\Psi_{p}(j)=\Psi_{0}-j \Psi_{1}$ be unitary, that is,

$$
\begin{aligned}
& \left\{\begin{array}{l}
\left(\Psi_{0}+\Psi_{1}\right)\left(\Psi_{0}^{T}+\Psi_{1}^{T}\right)=I_{M} \\
\left(\Psi_{0}-j \Psi_{1}\right)\left(\Psi_{0}^{T}+j \Psi_{1}^{T}\right)=I_{M}
\end{array}\right. \\
& \Leftrightarrow\left\{\begin{array}{l}
\Psi_{0} \Psi_{0}^{T}+\Psi_{1} \Psi_{1}^{T}+\Psi_{0} \Psi_{1}^{T}+\Psi_{1} \Psi_{0}^{T}=I_{M} \\
\Psi_{0} \Psi_{0}^{T}+\Psi_{1} \Psi_{1}^{T}+j\left(\Psi_{0} \Psi_{1}^{T}-\Psi_{1} \Psi_{0}^{T}\right)=I_{M}
\end{array}\right.
\end{aligned}
$$

Subtracting the two equations yields

$$
\begin{aligned}
& \Psi_{0} \Psi_{1}^{T}+\Psi_{1} \Psi_{0}^{T}-j\left(\Psi_{0} \Psi_{1}^{T}-\Psi_{1} \Psi_{0}^{T}\right)=0_{M} \\
& \quad \Leftrightarrow \Psi_{0} \Psi_{1}^{T}+\Psi_{1} \Psi_{0}^{T}=0_{M} \text { and } \Psi_{0} \Psi_{1}^{T}-\Psi_{1} \Psi_{0}^{T}=0_{M} \\
& \quad \Leftrightarrow \Psi_{0} \Psi_{1}^{T}=0_{M} \text { and } \Psi_{1} \Psi_{0}^{T}=0_{M} .
\end{aligned}
$$

Inserting into (17) yields $\Psi_{0} \Psi_{0}^{T}+\Psi_{1} \Psi_{1}^{T}=I_{M}$; all requirements (9a)-(9b) for a paraunitary $\Psi_{p}(z)$ are satisfied.

Lemma 2 chooses 1 and $j$ as evaluation points. Using a very similar proof, we can generalize to arbitrary roots of unity $\omega_{1}$ and $\omega_{2}$, provided $\omega_{2} \neq \pm \omega_{1}, \pm \omega_{1}^{*}$.

As an example application of Lemma 2, consider the $K \times K$ polyphase matrix

$\operatorname{DFT}_{p, K}(z)=\frac{1}{\sqrt{K}}\left[\cos \frac{2 k \ell \pi}{K}+z^{-1} \sin \frac{2 k \ell \pi}{K}\right]_{0 \leq k, \ell \leq K-1}$.

Both $\operatorname{DFT}_{p, K}(j)=\operatorname{DFT}_{K}$ and $\operatorname{DFT}_{p, K}(1)=\operatorname{DHT}_{K}$ (the discrete Hartley transform [22]) are unitary; hence, by Lemma 2, $\operatorname{DFT}_{p, K}(z)$ is paraunitary.

In Theorem 2 (the proof is in Appendix A), we show that specific submatrices of $\mathrm{DFT}_{p, K}(z)$ are paraunitary, and thus correspond to LOTs. In Section III-B we will seed these matrices to obtain LTFTs (this algorithm is depicted in Fig. 3).

Theorem 2: Let $\Psi_{p}(z)$ be an $M \times M$ submatrix of $\sqrt{K / M}$. $\operatorname{DFT}_{p, K}(z), K \geq M \geq 2$, constructed by selecting the following row and column sets:

$$
\begin{aligned}
\text { rows : } & \{r+k R \bmod K \mid 0 \leq k \leq M-1\} \\
\text { columns : } & \{c+\ell C \bmod K \mid 0 \leq \ell \leq M-1\}
\end{aligned}
$$

for some constants $0 \leq r, c, R, C<K$.

Then, $\Psi_{p}(z)$ is paraunitary if $K=M \operatorname{gcd}(K, R C)$ (in particular, $M$ divides $K$ ) and one of the following is satisfied:

i) $K$ divides $2 r C, 4 r c$, and $2 M R c$;

ii) $K$ does not divide $2 r C$, and $K$ divides both $2 r(2 c+$ $C M-C)$ and $R(2 c+C M-C)$.

Note that Theorem 2 implies that $M$ must divide $K$.

Since $\operatorname{DFT}_{p, K}(z)$ is symmetric, we can interchange the row and column index sets in the theorem.

Corollary 1: $\Psi_{p}(z)$ constructed as in Theorem 2 is paraunitary if and only if $\Psi_{p}(z)^{T}$ is paraunitary.

Note that in Theorem 2 we work with index sets instead of lists since permutations of rows and columns preserve paraunitarity.
Each paraunitary matrix $\Psi_{p}(z)$ obtained with Theorem 2 defines a basis $\Psi$; the associated LOT is $\Psi^{*}$.

We provide examples and further analysis in Section IV. Here, we first complete the theory and discuss the seeding of LTFTs from the above LOTs.

\section{B. Construction of New LTFTs From LOTs}

In this section, we seed $M \times M$ LOT matrices $\Psi_{p}(z)$, constructed as in Theorem 2, to obtain $N \times M$ frames $\Phi_{p}(z)$ and establish their properties.

Tightness: Any seeding of a $\Psi_{p}(z)$ obtained with Theorem 2 yields a tight frame $\Phi_{p}(z)$ by Lemma 1 .

Equal Norm: Every element of $\Psi_{p}(z)$ constructed with Theorem 2 has the norm $1 / \sqrt{M}$. Hence, the columns of any seeded $N \times M$ matrix $\Phi_{p}(z)$ have the same norm $\sqrt{N / M}$.

Maximally Robust Frames: In general, maximal robustness for frames is a property difficult to prove since one has to check that every $N \times N$ submatrix of $\Phi_{p}(z)$ is invertible. The good news is that it is sufficient to ensure that each such submatrix is nonsingular for at least one value [23].

Lemma 3: A square polyphase matrix $A_{p}(z)$ is nonsingular if and only if there exists $z_{0} \in \mathbb{C}$ such that $\operatorname{det} A_{p}\left(z_{0}\right) \neq 0$.

We will use this fact in the proof of the following theorem.

Theorem 3: Let $\Psi_{p}(z)$ be a paraunitary polyphase matrix constructed using Theorem 2 such that $M$ and $M R C / K$ are co-prime. Further, we seed a frame

$$
\Phi_{p}(z)=\Psi_{p}(z)[\mathcal{I}]
$$

by retaining $N<M$ rows. Then $\Phi_{p}(z)$ is maximally robust to erasures if (as sets)

$$
\mathcal{I}=\{d+D k \bmod M \mid 0 \leq k<N\}
$$

for some $0 \leq d<M$ and $D \equiv(M R C / K)^{-1} \bmod M$.

The proof is given in Appendix $B$.

As an example, consider the following family of maximally robust LTFTs.

Corollary 2: If $\Psi_{p}(z)$ is constructed as in Theorem 2 with $R=1, C=K / M$, and $r=c=0$, then any consecutive seeding (retaining of consecutive rows) of $\Psi_{p}(z)$ yields a maximally robust LTFT $\Phi_{p}(z)$.

Note that the LTFTs constructed as in Corollary 2 and seeded starting with the first row (i.e., $\mathcal{I}=\{0,1, \ldots, M-1\}$ ) are Weyl-Heisenberg frames [14].

\section{NEW LOTS AND LTFTS: EXAMPLES AND ANALYSIS}

In this section, we construct new classes of real LOTs and LTFTs using the theory from Section III. We first provide examples of LOTs constructed with Theorem 2; we also show that the previously known real LOTs (10)-(11) can be constructed using Theorem 2. Then, we seed these LOTs to construct LTFTs. Further, we discuss the design of windows for the LTFTs, providing additional flexibility in design. Finally, we briefly discuss complex LTFTs constructed from the complex LOTs (12)-(13). Note that, as before, we always construct bases $\Psi$ and frames $\Phi$; the associated LOTs and LTFTs are given by $\Psi^{*}$ and $\Phi^{*}$. 


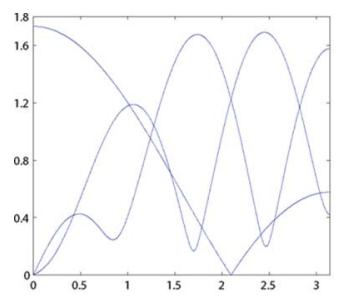

(a)

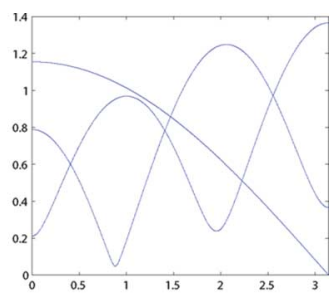

(b)
Fig. 4. Magnitude responses of (a) LOT basis vectors in (19) and (b) seeded LTFT frame vectors in (21) derived using our construction method. The corresponding filter bank has $M=3$ filters with support $2 M=6$ (LOT) or $2 N=4$ (LTFT).

\section{A. New LOTs}

Small Example: We start with $\operatorname{DFT}_{p, 6}(z)$ and construct a polyphase matrix using Theorem 2 with parameters $K=6, M=3, R=4, C=1, r=0, c=0$ and get

$$
\Psi_{p}(z)=\frac{1}{\sqrt{3}}\left(\begin{array}{ccc}
1 & 1 & 1 \\
1 & -\frac{1}{2}-\frac{\sqrt{3}}{2} z^{-1} & -\frac{1}{2}+\frac{\sqrt{3}}{2} z^{-1} \\
1 & -\frac{1}{2}+\frac{\sqrt{3}}{2} z^{-1} & -\frac{1}{2}-\frac{\sqrt{3}}{2} z^{-1}
\end{array}\right)
$$

Since the parameters satisfy condition (i) of the theorem, $\Psi_{p}(z)$ is paraunitary and hence specifies an LOT.

Fig. 4(a) depicts the magnitude responses of the basis vectors in this example.

Class of LOTs: Next we give one example of how to construct an entire class of LOTs for any size $M$. In Theorem 2, we choose $K=a M$ with any $a \in \mathbb{N}, r=c=0, R=1$ and $C=a$ to satisfy condition (i). Then, for any $a \in \mathbb{N}$,

$$
\Psi_{p, a}(z)=\frac{1}{\sqrt{M}}\left[\cos \frac{2 k \ell a \pi}{K}+\sin \frac{2 k \ell a \pi}{K} z^{-1}\right]_{0 \leq k, \ell<M}
$$

is paraunitary, that is, $\Psi^{*}$ is an LOT.

Number of New LOTs: We now investigate how many $M \times$ $M \Psi_{p}(z)$ can be derived from $\operatorname{DFT}_{p, K}(z)$ and Theorem 2 . Necessarily, $M \mid K$, which implies that $K$ is not prime. This in mind, Table I shows the number of new LOTs generated using our method. For example, there are $283 \times 3$ paraunitary submatrices of $\operatorname{DFT}_{p, 6}(z)$ and all are found with the theorem. Note that every submatrix is specified by a row subset and column subset of $\operatorname{DFT}_{p, 6}(z)$; the ordering does not matter.

Further, there are $405 \times 5$ paraunitary submatrices of $\mathrm{DFT}_{p, 10}$ that do not arise from Theorem 2. One such example is the row set $\{0,1,3,7,9\}$, and the column set $\{0,2,4,6,8\}$. However, we speculate that these matrices are up to permutations the same as other submatrices that are derived from the theorem. In fact, Theorem 2 could be extended based on the permutation symmetries of the DFT [24], [25] and may then cover all paraunitary submatrices.

Finally, we must note that empirical tests show that there are no $M \times M$ paraunitary submatrices of $\mathrm{DFT}_{p, K}(z)$ for $M$ not dividing $K$, for $K \leq 16$.
TABLE I

Number of PARAunitary $M \times M \Psi_{p}(z)$ GENERATED From DFT ${ }_{p, K}$ Using THEOREM 2. THE NuMBERS OF PARAUNITARY SUBMATRICES $\Psi_{p}(z)$ That Do Not SATISFy ThEOREM 2 ARE ShOWn IN ITALIC

\begin{tabular}{rrrrrrrr}
\hline & \multicolumn{7}{c}{$M$} \\
\cline { 2 - 7 }$K$ & 2 & 3 & 4 & 5 & 6 & 7 & 8 \\
\hline 4 & 16 & & & & & & \\
6 & 17 & 28 & & & & & \\
8 & 128 & - & 64 & & & & \\
9 & - & 66 & & & & & \\
10 & 49 & - & - & 84 & & & \\
12 & 304 & 384 & 53 & - & 96 & & \\
14 & 97 & - & - & - & - & 172 & \\
15 & - & 161 & - & 141 & & & \\
16 & 896 & - & 1216 & - & - & - & 256 \\
& & & 1088 & & & & 768 \\
\hline
\end{tabular}

\section{B. New Real LTFTs}

Small Example: As a small example we seed (19) by retaining the first two rows and get

$$
\begin{aligned}
\Phi_{p}(z) & =\frac{1}{\sqrt{3}}\left(\begin{array}{ccc}
1 & 1 & 1 \\
1 & -\frac{1}{2}-\frac{\sqrt{3}}{2} z^{-1} & -\frac{1}{2}+\frac{\sqrt{3}}{2} z^{-1}
\end{array}\right) \\
& =\frac{1}{\sqrt{3}}\left(\begin{array}{ccc}
1 & 1 & 1 \\
1 & -\frac{1}{2} & -\frac{1}{2}
\end{array}\right)+\frac{1}{\sqrt{3}} z^{-1}\left(\begin{array}{ccc}
0 & 0 & 0 \\
0 & -\frac{\sqrt{3}}{2} & \frac{\sqrt{3}}{2}
\end{array}\right) \\
& =\Phi_{0}+z^{-1} \Phi_{1} .
\end{aligned}
$$

By construction, this frame is tight and equal norm. By Theorem 3, any seeding at stride $(M R C / K)^{-1} \equiv 1 \bmod M$ (that is, consecutive) yields a maximally robust frame; hence, the constructed LTFT is also maximally robust.

The frame $\Phi$ has columns $\left\{\varphi_{3 k}, \varphi_{3 k+1}, \varphi_{3 k+2}\right\}_{k \in \mathbb{Z}}$ with

$$
\begin{aligned}
\varphi_{3 k} & =\frac{1}{\sqrt{3}}(\ldots, 1,1,0,0, \ldots)^{T} \\
\varphi_{3 k+1} & =\frac{1}{\sqrt{3}}\left(\ldots, 1,-\frac{1}{2}, 0,-\frac{\sqrt{3}}{2}, \ldots\right)^{T} \\
\varphi_{3 k+2} & =\frac{1}{\sqrt{3}}\left(\ldots, 1,-\frac{1}{2}, 0, \frac{\sqrt{3}}{2}, \ldots\right)^{T}
\end{aligned}
$$

where the first element 1 in each vector is at position $k$.

$\Phi$ can be viewed as a lapped counterpart of the MercedesBenz frame in (15). Fig. 4(b) depicts the magnitude responses of the frame vectors in this example.

Class of LTFTs: Given any $N<M$, an $N \times M \Phi_{p}(z)$ can be constructed by seeding the $M \times M \Psi_{p}(z)$ in (20), retaining $N$ rows. Any such frame will be tight and equal norm. Since the construction parameters satisfy Theorem 3, and $(M R C / K)^{-1} \equiv 1 \bmod M, \Phi_{p}(z)$ also is maximally robust if it results from consecutive seeding. 


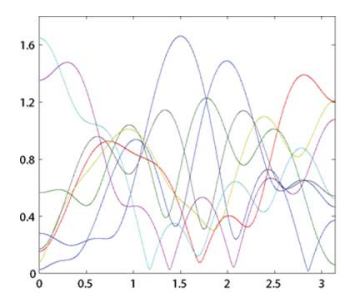

(a)

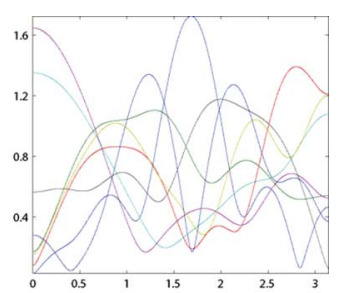

(b)
Fig. 5. Magnitude responses of LTFT frame vectors resulting from consecutive seeding with $M=8, N=5$ of the (a) Princen-Johnson-Bradley and (b) oddlymodulated DCT LOTs. In each case the corresponding filter bank has $M=8$ filters of support $2 N=10$.

\section{Known Real LOTs and LTFTs}

We demonstrate that the two known families of real LOTs (Princen-Johnson-Bradley and Oddly-Modulated DCT) in (10) and (11) can be derived using Theorem 2.

First, we compute the corresponding polyphase matrices. Note that both (10) and (11) show $\left[\Psi_{0}^{T} \Psi_{1}^{T}\right]$ and we have to compute $\Psi_{p}(z)=\Psi_{0}+z^{-1} \Psi_{1}$. Below, the row index is $k$ and the column index is $m$.

For the Princen-Johnson-Bradley LOT (10),

$$
\Psi_{p}(z)=\frac{1}{\sqrt{M}}\left[\cos \alpha_{k, m}+z^{-1} \sin \alpha_{k, m}\right]
$$

where $\alpha_{k, m}=(-1)^{m+1}(2 m+1)(2 k+1-M) \pi / 4 M$.

For the Oddly-Modulated DCT LOT (11),

$$
\Psi_{p}(z)=\frac{1}{\sqrt{M}}\left[\cos \beta_{k, m}+z^{-1} \sin \beta_{k, m}\right]
$$

where $\beta_{k, m}=(-1)^{m+1}(2 m+1)(2 k+1+M) \pi / 4 M$.

In both cases, $\Psi_{p}^{T}(z)$ can be constructed as in Theorem 2 with parameters $K=8 M, R=2, C=4, r=7 M+1, c=$ $6 M+2+(-1)^{M}$ for the Princen-Johnson-Bradley LOT; and $K=8 M, R=2, C=4, r=M+1, c=6 M+2+(-1)^{M}$ for the Oddly-Modulated DCT LOT. These parameters satisfy condition ii) of the theorem, and thus $\Psi_{p}(z)^{T}$ is paraunitary. By Corollary $1, \Psi_{p}(z)$ is paraunitary as well.

As a consequence, any frame $\Phi_{p}(z)$ seeded from the above $\Psi_{p}(z)$ is tight and equal-norm.

Finally, using Theorem 3 we conclude that any consecutive seeding of the above $\Psi_{p}^{T}(z)$ yields a maximally robust LTFT, with vector norm $\sqrt{N / M}$.

As an example, Fig. 5(a) and (b) shows the magnitude responses of the frame vectors constructed by preserving the first $N=5$ rows of $\Psi_{p}(z)$ of size $M=8$ for the Princen-JohnsonBradley and the Oddly-Modulated DCT LOTs, respectively.

\section{Window Design}

We can gain additional freedom in LTFT construction, as well as improve the frequency behavior of the corresponding filters in the filter bank, by windowing the constructed LTFTs.

We are interested in one window that modulates all frame functions at once. That is, we seek to design a $2 N \times 2 N$ diagonal window matrix $\Delta=\operatorname{diag}\left(\delta_{0}, \ldots, \delta_{2 N-1}\right)$ that produces a windowed LTFT

$$
\Phi_{\Delta}=\Delta \cdot\left(\begin{array}{l}
\Phi_{0} \\
\Phi_{1}
\end{array}\right)
$$

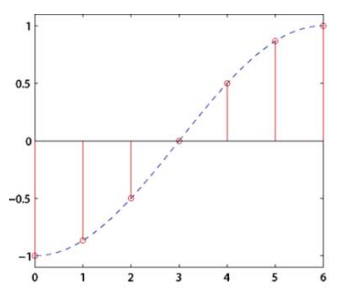

(a)

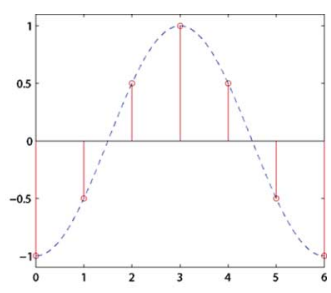

(b)
Fig. 6. Window solution to (25a)-(25b) for (a) $N=7$ and (b) $N=8$.

We use two different approaches to the window design. The first one is analytical and uses algebraic conditions that govern the filter bank. The second one uses optimization techniques to approximate the frequency behavior of HTFs, as these are narrow bandpass filters evenly spread across the frequency domain.

The following derivations apply to all LTFTs seeded from LOTs. As a running example, we use LTFTs seeded from the Princen-Johnson-Bradley LOT (10).

Analytical Approach: For a LTFT seeded from the PrincenJohnson-Bradley LOT, the $(k, m)$ th element of $\Phi_{0} \Phi_{0}^{*}$ or $\Phi_{1} \Phi_{1}^{*}$ is

$a_{k, m}=\frac{1}{4 M} \frac{\sin (\pi(k-m))}{\sin \left(\frac{\pi(k-m)}{2 M}\right)}+(-1)^{M+1} \frac{1}{4 M} \frac{\sin (\pi(k+m+1))}{\cos \left(\frac{\pi(k+m+1)}{2 M}\right)}$,

for $k, m=0, \ldots, N-1$.

Substituting (24) into (4), we get the following system of equations:

$$
\begin{aligned}
a_{m, m} \delta_{m}^{2}+\left(1-a_{m, m}\right) \delta_{N+m}^{2} & =1, \\
\delta_{N+m} \delta_{N+k} & =\delta_{m} \delta_{k},
\end{aligned}
$$

for $0 \leq k, m<N, k \neq m$. It has an infinite set of solutions. Among them let us consider symmetric ones: $\delta_{m}=\delta_{2 M-1-m}$. In this case, as shown in Appendix $\mathrm{C}$, we obtain the following system of equations:

$$
\begin{aligned}
a_{m, m} \delta_{m}^{2}+\left(1-a_{m, m}\right) \delta_{N-m-k}^{2} & =1, \\
\delta_{N-m-1} \delta_{N-k-1} & =\delta_{m} \delta_{k},
\end{aligned}
$$

for $0 \leq k, m<N, k \neq m$. Fixing $\delta_{0}=-1$, we have $\delta_{N-1}=$ \pm 1 and $\delta_{k}=-\delta_{N-1} \delta_{N-k-1}$ for $1 \leq k<N-1$.

An example of a symmetric window is shown in Fig. 6. It is given by

$$
\delta_{m}=\left\{\begin{array}{ll}
\cos \left(\frac{m \pi}{N-1}+\pi\right) & \text { for } N \text { even, } \\
\cos \left(\frac{2 m \pi}{N-1}+\pi\right) & \text { for } N \text { odd, }
\end{array} \quad 0 \leq M<N .\right.
$$

Also, note that conditions (25a)-(25b) hold for anti-symmetric windows, for which $\delta_{m}=-\delta_{2 M-1-m}$.

Optimization Approach: We first explore error minimization algorithms. The procedure finds an optimal window $\hat{\delta}$ that minimizes the weighted error between an HTF and the frame seeded from the Princen-Johnson-Bradley LOT in the frequency domain as follows:

$$
\hat{\delta}=\underset{\delta}{\operatorname{argmin}} \kappa \odot\left(\Phi^{(\mathrm{HTF})}-\delta \circledast \Phi^{(\mathrm{PJB})}\right)
$$




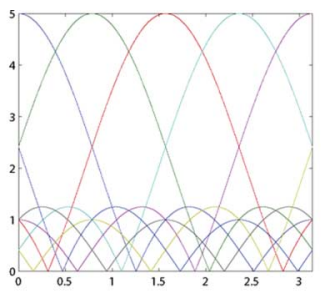

(a)

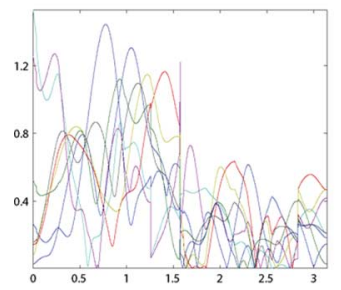

(c)

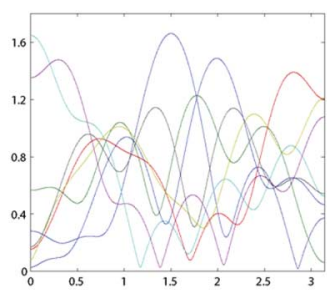

(b)

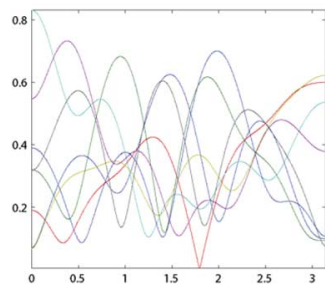

(d)

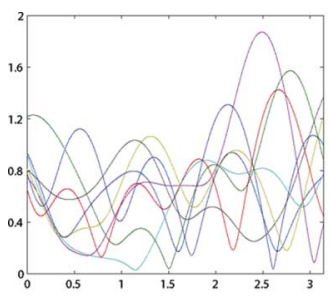

(a)

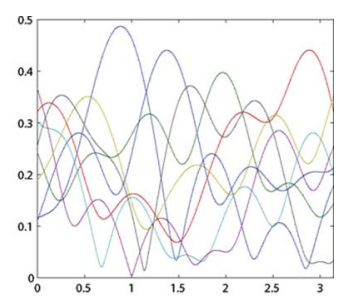

(b)

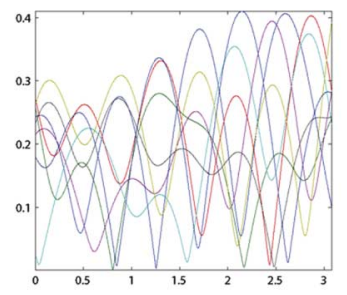

(c)

Fig. 8. Magnitude responses of the windowed Princen-Johnson-Bradley LTFT frame vectors using the polar decomposition method with $M=8, N=5$, and (a) $\hat{\Delta}_{1}$, (b) $\hat{\delta}_{2}$, and (c) $\hat{\delta}_{3}$.

for any unitarily invariant norm. Thus, by taking $\Theta=$ $\Phi^{(\mathrm{HTF})} \Phi^{(\mathrm{PJB})^{*}}$ and $\Delta$ the window matrix, we have

$$
\hat{\Delta}=\underset{\Delta}{\operatorname{argmin}}\left\|\Delta-\Phi^{(\mathrm{HTF})} \Phi^{(\mathrm{PJB})^{*}}\right\| .
$$

Ideally, we would like to have one window vector for the entire set of LTFT filters (that is, we want $\hat{\Delta}$ to be diagonal). Hence, we can use $\hat{\Delta}$ in three different ways:

1) $\hat{\Delta}_{1}=\hat{\Delta}$; each column of $\hat{\Delta}_{1}$ windows one LTFT filter;

2) $\hat{\delta}_{2}$ is the vector of eigenvalues of $\hat{\Delta} ; \hat{\delta}_{2}$ windows all LTFT filters;

3) $\hat{\delta}_{3}=\lambda e$, with $\lambda$ the largest eigenvalue, $e$ the corresponding eigenvector of $\hat{\Delta} ; \hat{\delta}_{3}$ windows all LTFT filters.

Fig. 8 shows the magnitude responses of the PrincenJohnson-Bradley LTFT filters when windowed by $\hat{\Delta}_{1}, \hat{\delta}_{2}$ and $\hat{\delta}_{3}$, showing a slight improvement over the original Princen-Johnson-Bradley LTFT in that they have a better frequency localization, with $\hat{\delta}_{2}$ being the best one.

\section{E. Complex LTFTs}

Complex LOTs, such as (12) and (13), do not fit our current framework since one cannot apply Lemma 2 . However, we postulate that there could be a more general construction method involving complex matrices, the study of which we leave for future work.

Here, we briefly consider seeding the complex bases underlying the LOTs (12) and (13). Because of Lemma 1, any seeding yields a tight frame. In addition, any such frame is equal norm. To show this, we first compute the respective polyphase matrices. Note that both (12) and (13) show $\left[\Psi_{0}^{*} \Psi_{1}^{*}\right]$ and we have to compute $\Psi_{p}(z)=\Psi_{0}+z^{-1} \Psi_{1}$. Below, the row index is $k$, the column index is $m$, and $\alpha_{k}=(2 k+1) \pi /(4 M)$.

For the Young-Kingsbury LOT (12), $\Psi_{p}(z)$ is given by

$$
-\frac{1}{\sqrt{M}}\left[\omega_{8 M}^{-(2 k+1)(2 m+1)}\left((-1)^{m} j \sin \alpha_{k}-z^{-1} \cos \alpha_{k}\right)\right],
$$

$$
\|\Delta-\Theta\|=\min \left\{\|\Delta-Q\|: Q^{*} Q=I_{2 N}\right\}
$$




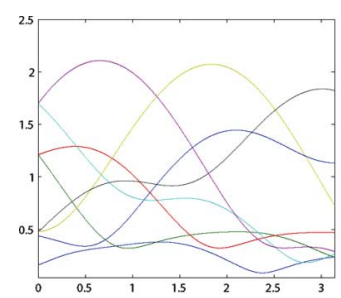

(a)

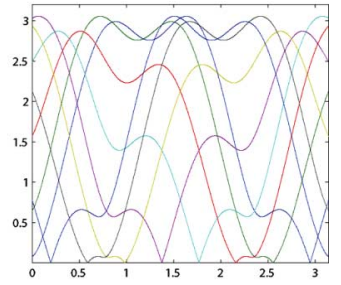

(b)
Fig. 9. Magnitude responses of LTFT frame vectors resulting from consecutive seeding with $M=8, N=5$ of the (a) Young-Kingsbury and (b) Malvar LOTs. In each case the corresponding filter bank has $M=8$ filters of support $2 N=10$.

and for the Malvar LOT (13), $\Psi_{p}(z)$ is given by

$$
-\sqrt{\frac{2}{M}}\left[\omega_{8 M}^{(2 k+1+M)(2 m+1)}\left(\sin \alpha_{k}-(-1)^{m} z^{-1} j \cos \alpha_{k}\right)\right] .
$$

Observe that in both polyphase matrices every entry has the same norm, $\sqrt{1 / M}$ and $\sqrt{2 / M}$, respectively. Hence, every seeded frame will be equal norm.

As an example, we consider $M=8$ and seed in both cases by retaining the first 5 rows $(N=5)$ to obtain an equal-norm tight frame. The magnitude responses of the associated analysis filters for the Young-Kingsbury frame are shown in Fig. 9(a), and for the Malvar frame are shown in Fig. 9(b).

\section{CONCLUSIONS AND FUTURE WORK}

We presented a simple and flexible construction method to generate new LOTs from DFTs and new LTFTs from these new LOTs. The new LTFTs are tight, equal-norm and under certain conditions, maximally robust to erasures. We have shown that some well-known LOTs can be seen as particular cases constructed with our method, and have studied LTFTs derived from these LOTs. In addition, although excluded from the current framework, we discussed known complex LOT families and showed that they lead to equal-norm, tight LTFTs.

We intend to extend our construction method to extended lapped transforms for which the length $L$ of the filters is any integer multiple $q \geq 2$ of $M$ (in which case $\Psi_{p}(z)$ contains polynomials of degree $q-1$ ). Moreover, we plan on generalizing the method to the complex case, by investigating the complex counterparts to Lemma 2 and Theorem 2. For example, we have seen that, for $q=2$, the equivalence between unitary and paraunitary matrices relies on the pair $(1, j)$, or, more generally, on any two roots of unity with an angle between them that differs from $\pi$ and $\pi / 2$. Do $q$-tuplets of complex numbers then exist, such that the same equivalence is preserved in the case where $q>2$ ? Another interesting venue would be to generalize our construction method to include a larger class of LOTs and LTFTs, and to find the most general sufficient and necessary conditions on the design of paraunitary submatrices of the DFT.

\section{APPENDIX A}

\section{PROOF OF THEOREM 2}

According to Lemma 2, to show that $\Psi_{p}(z)$ is paraunitary, it is enough to show that $\Psi_{p}(j)$ and $\Psi_{p}(1)$ are unitary.
The elements of the matrix $\Psi_{p}(z)$ are

$$
\begin{aligned}
\psi_{k, \ell}(z)=\frac{1}{\sqrt{M}}(\cos ( & \left.\frac{2 \pi(r+k R)(c+\ell C)}{K}\right) \\
& \left.+\sin \left(\frac{2 \pi(r+k R)(c+\ell C)}{K}\right) z^{-1}\right),
\end{aligned}
$$

$0 \leq k, \ell \leq M-1$ and $0 \leq r, c, R, C \leq M-1$.

We first find the conditions for $\Psi_{p}(j)$ to be unitary. The $(k, \ell)$ th element of $\Psi_{p}(j) \Psi_{p}^{*}(j)$ is given by

$$
\begin{aligned}
\left(\Psi_{p}(j) \Psi_{p}^{*}(j)\right)_{k, \ell} & =\frac{1}{M} \sum_{m=0}^{M-1} \omega_{K}^{(r+k R)(c+m C)-(r+\ell R)(c+m C)} \\
& =\frac{1}{M} \omega_{K}^{(k-\ell) R c} \sum_{m=0}^{M-1} \omega_{K}^{(k-\ell) R C m} \\
& = \begin{cases}1, & k=\ell ; \\
\frac{1}{M} \omega_{K}^{(k-\ell) R c} \frac{1-\omega_{K}^{(k-\ell) R C M}}{1-\omega_{K}^{(k-\ell) R C}}, & k \neq \ell .\end{cases}
\end{aligned}
$$

$\Psi_{p}(j)$ is unitary if and only if $\left(\Psi_{p}(j) \Psi_{p}^{*}(j)\right)_{k, \ell}=0$ for any $k \neq \ell$, or, equivalently, if and only if $K$ is divisible by the product $R C M$, but not divisible by $(k-\ell) R C$ for any $k-\ell \neq 0$ such that $1 \leq|k-\ell| \leq M-1$. This is possible if and only if $K=M \operatorname{gcd}(K, R C)$. Thus, $\Psi_{p}(j) \Psi_{p}^{*}(j)=I_{M}$, and $\Psi_{p}(j)$ is unitary if and only if $K=M \operatorname{gcd}(K, R C)$.

We next investigate conditions for $\Psi_{p}(1)$ to be unitary. The $(m, \ell)$ th element of $\Psi_{p}(1)$ is

$$
\begin{aligned}
\psi_{k, \ell}(1)= & \frac{1}{\sqrt{M}}\left(\cos \left(\frac{2 \pi(r+k R)(c+\ell C)}{K}\right)\right. \\
& \left.+\sin \left(\frac{2 \pi(r+k R)(c+\ell C)}{K}\right)\right) \\
= & \frac{1}{\sqrt{M}}\left(\frac{1+j}{2} \omega_{K}^{(r+k R)(c+\ell C)}\right. \\
& \left.+\frac{1-j}{2} \omega_{K}^{-(r+k R)(c+\ell C)}\right) .
\end{aligned}
$$

The $(k, \ell)$ th element of $\Psi_{p}(1) \Psi_{p}^{*}(1)$ is

$$
\begin{aligned}
&\left(\Psi_{p}(1) \Psi_{p}^{*}(1)\right)_{k, \ell} \\
&=\frac{1}{M} \sum_{m=0}^{M-1}[\left(\frac{1+j}{2} \omega_{K}^{(r+k R)(c+m C)}\right. \\
&\left.+\frac{1-j}{2} \omega_{K}^{-(r+k R)(c+m C)}\right) \\
& \times\left(\frac{1-j}{2} \omega_{K}^{-(r+\ell R)(c+m C)}\right. \\
&\left.\left.+\frac{1+j}{2} \omega_{K}^{(r+\ell R)(c+m C)}\right)\right] \\
&=\frac{1}{2 M} \sum_{m=0}^{M-1}\left(\omega_{K}^{(k-\ell) R(c+k C)}+\omega_{K}^{(\ell-k) R(c+m C)}\right) j \sum_{m=0}^{M-1}\left(\omega_{K}^{(2 r+(k+\ell) R)(c+m C)}\right. \\
&\left.+\frac{j}{2 M} \sum_{m}^{-(2 r+(k+\ell) R)(c+m C)}\right) \\
&=\frac{j}{2 M} \sum_{k, \ell}^{(1)}+\frac{j}{2 M} \Sigma_{k, \ell}^{(2)} .
\end{aligned}
$$


Since $K=M \operatorname{gcd}(K, R C)$, then for any $0 \leq k, \ell \leq M-1$ with $k \neq \ell, K$ is not divisible by $(k-\ell) R C$. Thus,

$$
\begin{aligned}
\Sigma_{k, \ell}^{(1)} & = \begin{cases}\sum_{m=0}^{M-1} 2, & k=\ell ; \\
\omega_{K}^{(k-\ell) R c} \frac{1-\omega_{K}^{(k-\ell) R C M}}{1-\omega_{K}^{(k-\ell) R C}} & \\
+\omega_{K}^{(\ell-k) R c} \frac{1-\omega_{K}^{(\ell-k) R C M}}{1-\omega_{K}^{(\ell-k) R C}}, & k \neq \ell ;\end{cases} \\
& = \begin{cases}2 M, & k=\ell ; \\
0, & k \neq \ell .\end{cases}
\end{aligned}
$$

To make $\Psi_{p}(1)$ a unitary matrix, we choose to impose the condition $\Sigma_{k, \ell}^{(2)}=0$ for any $0 \leq k, \ell \leq M-1$. Here, we consider the two cases specified by the theorem.

Case (i): If $K$ divides $2 r C, 4 r c$, and $2 M R c$, then for any $k, \ell$

$$
\begin{aligned}
\Sigma_{k, \ell}^{(2)}= & \omega_{K}^{(2 r+(k+\ell) R) c} \sum_{m=0}^{M-1} \omega_{K}^{(2 r+(k+\ell) R) C m} \\
& -\omega_{K}^{-(2 r+(k+\ell) R) c} \sum_{m=0}^{M-1} \omega_{K}^{-(2 r+(k+\ell) R) C m} \\
= & \begin{cases}M\left(\omega_{K}^{2 r c}-\omega_{K}^{-2 r c}\right), & k+\ell=0 ; \\
M\left(\omega_{K}^{2 r c+M R c}-\omega_{K}^{-2 r c-M R c}\right), & k+\ell=M ; \\
\omega_{K}^{2 r c+(k+\ell) R c} \frac{1-\omega_{K}^{(k+\ell) R C M}}{1-\omega_{K}^{(k+\ell) R C}} & \\
-\omega_{K}^{-2 r c-(k+\ell) R c_{0}} \frac{1-\omega_{K}^{-(k+\ell) R C M}}{1-\omega_{K}^{-(k+\ell) R C}}, & \text { otherwise; } \\
= & 0 .\end{cases}
\end{aligned}
$$

Case (ii): If $K$ does not divide $2 r C$, then $\Sigma_{k, \ell}^{(2)}=0$ is equivalent to

$$
\omega_{K}^{(2 r+(k+\ell) R)(2 c+C M-C)}=1,
$$

for any $k, \ell$. This is possible if $K$ divides both $2 r(2 c+C M-C)$ and $R(2 c+C M-C)$.

Thus, in either of the two cases $\Psi_{p}(1) \Psi_{p}^{*}(1)=I_{M}$, and $\Psi_{p}(1)$ is unitary.

Since the above conditions make $\Psi_{p}(j)$ and $\Psi_{p}(1)$ unitary, Lemma 2 implies that $\Psi_{p}(z)$ is paraunitary.

\section{APPENDIX B}

\section{PROOF OF THEOREM 3}

We use Lemma 3 with $z_{0}=j$, which makes $\Psi_{p}(j)$ a submatrix of $\sqrt{K / M} \mathrm{DFT}_{K}$. We fix the order of rows and columns and get

$$
\begin{aligned}
\Psi_{p}(j) & =\frac{1}{\sqrt{M}}\left[\omega_{K}^{(r+k R)(c+\ell C)}\right]_{0 \leq k, \ell<M} \\
& =\frac{1}{\sqrt{M}}\left[\omega_{K}^{r c} \cdot \omega_{K}^{c k R} \cdot \omega_{K}^{r \ell C} \cdot \omega_{K}^{k \ell R C}\right]_{0 \leq k, \ell<M} \\
& =\frac{1}{\sqrt{M}} \omega_{K}^{r c} \cdot \Omega_{1} \cdot\left[\omega_{K}^{k \ell R C}\right]_{0 \leq k, \ell<M} \cdot \Omega_{2} .
\end{aligned}
$$

Here, $\Omega_{1}=\operatorname{diag}\left(\omega_{K}^{c k R}\right)_{0 \leq k<M}$ and $\Omega_{2}=\operatorname{diag}\left(\omega_{K}^{r \ell C}\right)_{0 \leq \ell<M}$ are full-rank diagonal matrices, and $\omega_{K}^{r c} \neq 0$. Hence, by Proposition 1(iii) we can omit them in studying the seeding of MR frames.
Setting $M R C / K=A$ yields

$$
\omega_{K}^{k \ell R C}=\omega_{K}^{k \ell \frac{A K}{M}}=\left(\omega_{M}^{A}\right)^{k \ell} .
$$

Since $\operatorname{gcd}(M, A)=1, \omega_{M}^{A}$ is a primitive $M$ th root of unity, and thus

$$
\frac{1}{\sqrt{M}}\left[\omega_{K}^{k \ell R C}\right]_{0 \leq k, \ell \leq M-1}=P \cdot \mathrm{DFT}_{M} \cdot P^{T}
$$

where $P$ is the $M \times M$ permutation matrix:

$$
P_{k \ell}= \begin{cases}1, & \text { if } \ell=A k \bmod M \\ 0, & \text { otherwise. }\end{cases}
$$

Further, let $D \equiv(M R C / K)^{-1} \bmod M, 1 \leq D<M$, and consider an $N \times M$ submatrix of (29), constructed by selecting rows $\mathcal{I}=\{d+D k \bmod M \mid 0 \leq k<N\}$. Then

$$
\left(P \cdot \operatorname{DFT}_{M} \cdot P^{T}\right)[\mathcal{I}]=\operatorname{DFT}_{M}[\mathcal{J}] \cdot P^{T}
$$

where $\mathcal{J}=\{d A+k \bmod M \mid 0 \leq k<N\}$. Since $\operatorname{DFT}_{M}[\mathcal{J}]$ is an $N \times M$ submatrix of $\mathrm{DFT}_{M}$ constructed from adjacent rows (possibly looping around the bottom of the matrix), each $N \times N$ submatrix of it is invertible [20]. It follows from Proposition 1 that each $N \times N$ submatrix of $\Phi_{p}(j)$ is also invertible.

Hence, by Lemma 3, every $N \times N$ submatrix of $\Phi_{p}(z)$ is nonsingular, and $\Phi_{p}(z)$ is maximally robust to erasures.

\section{APPENDIX C}

DERIVATION OF CONDITIONS (25a)-(25b) FOR THE SYMMETRIC WINDOW $\Delta$

For a symmetric window, we have

$$
\Delta=\left(\begin{array}{cc}
\Delta_{0} & \\
& J_{N} \Delta_{0} J_{N}
\end{array}\right)
$$

where $J_{N}$ is a $N \times N$ complimentary diagonal matrix: $J_{N}(k, m)=1$ if $k+m=N-1$ and 0 otherwise.

Substituting (24) into (4), we get

$$
\begin{aligned}
\Delta_{0} \Phi_{0} \Phi_{0}^{*} \Delta_{0}+J_{N} \Delta_{0} J_{N} \Phi_{1} \Phi_{1}^{*} J_{N} \Delta_{0} J_{N} & =I, \\
\Phi_{0} \Phi_{0}^{*}+\Phi_{1} \Phi_{1}^{*} & =I .
\end{aligned}
$$

Using $\Phi_{0} \Phi_{0}^{*}=I-\Phi_{1} \Phi_{1}^{*}$, we rewrite (32a) as

$$
\Delta_{0} \Phi_{0} \Phi_{0}^{*} \Delta_{0}+J_{N} \Delta_{0}^{2} J_{N}-J_{N} \Delta_{0} J_{N} \Phi_{0} \Phi_{0}^{*} J_{N} \Delta_{0} J_{N}=I
$$

where $\Delta_{0}=\operatorname{diag}\left\{\delta_{n}\right\}_{n=0}^{N-1}$.

If $\left(\Phi_{0} \Phi_{0}^{*}\right)_{k, m}=a_{k, m}$, then we get

$$
\begin{aligned}
& \Delta_{0} \Phi_{0} \Phi_{0}^{*} \Delta_{0} \\
& \quad=\left(\begin{array}{cccc}
\delta_{0}^{2} a_{0,0} & \ldots & \delta_{j} \delta_{0} a_{0, m} & \ldots \\
\delta_{0} \delta_{1} a_{1,0} & \ldots & \delta_{j} \delta_{1} a_{1, m} & \ldots \\
\delta_{0} \delta_{2} a_{2,0} & \ldots & \delta_{j} \delta_{2} a_{2, m} & \ldots \\
\vdots & \ddots & \vdots & \ldots \\
\delta_{0} \delta_{N-1} a_{N-1,0} & \ldots & \delta_{m} \delta_{N-1} a_{N-1, m} & \ldots
\end{array}\right)
\end{aligned}
$$


and

$$
\begin{aligned}
J_{N} \Delta_{0} J_{N} \Phi_{0} \Phi_{0}^{*} J_{N} \Delta_{0} J_{N} & \\
& =\left(\begin{array}{cccc}
\delta_{N-1}^{2} a_{0,0} & \ldots & \delta_{N-m-1} \delta_{N-1} a_{0, m} & \ldots \\
\delta_{N-1} \delta_{N-2} a_{1,0} & \ldots & \delta_{N-m-1} \delta_{N-2} a_{1, m} & \ldots \\
\delta_{N-1} \delta_{N-3} a_{2,0} & \ldots & \delta_{N-m-1} \delta_{N-3} a_{2, m} & \ldots \\
\vdots & \ddots & \vdots & \ldots \\
\delta_{N-1} \delta_{0} a_{N-1,0} & \ldots & \delta_{N-m-1} \delta_{0} a_{N-1, m} & \ldots
\end{array}\right) .
\end{aligned}
$$

It follows from (33) that $\delta_{m}$ have to satisfy

$$
\begin{aligned}
\left(\delta_{m}^{2}-\delta_{N-m-1}^{2}\right) a_{m, m}+\delta_{N-m-1}^{2} & =1 \\
\delta_{m} \delta_{k}-\delta_{N-m-1} \delta_{N-k-1} & =0
\end{aligned}
$$

for $0 \leq k, m \leq N-1, k \neq m$. These are exactly conditions (25a)-(25b).

\section{ACKNOWLEDGMENT}

The authors would like to thank D.-C. Balcan for helpful discussions on the polar decomposition.

\section{REFERENCES}

[1] R. J. Duffin and A. C. Schaeffer, "A class of nonharmonic Fourier series," Trans. Amer. Math. Soc., vol. 72, pp. 341-366, 1952.

[2] I. Daubechies, A. Grossman, and Y. Meyer, "Painless nonorthogonal expansions," J. Math. Phys., vol. 27, pp. 1271-1283, Nov. 1986.

[3] I. Daubechies, Ten Lectures on Wavelets. Philadelphia, PA: SIAM, 1992.

[4] J. Kovačević and A. Chebira, "An introduction to frames," Found. Trends Signal Process., vol. 2, no. 1, pp. 1-94, 2008.

[5] A. Chebira, Y. Barbotin, C. Jackson, T. E. Merryman, G. Srinivasa, R. F. Murphy, and J. Kovačević, "A multiresolution approach to automated classification of protein subcellular location images," BMC Bioinformat. vol. 8, no. 210, 2007 [Online]. Available: http://www.andrew.cmu.edu/_user/_jelenak/_Repository/07_ChebiraBJMSMK/07_ChebiraBJMSMK.html

[6] A. Chebira, J. A. Ozolek, C. A. Castro, W. G. Jenkinson, M. Gore, R. Bhagavatula, I. Khaimovich, S. E. Ormon, C. S. Navara, M. Sukhwani, K. E. Orwig, A. Ben-Yehudah, G. Schatten, G. K. Rohde, and J. Kovačević, "Multiresolution identification of germ layer components in teratomas derived from human and nonhuman primate embryonic stem cells," in Proc. IEEE Int. Symp. Biomed. Imaging, Paris, France, May 2008, pp. 979-982.

[7] R. A. Kellogg, A. Chebira, A. Goyal, P. A. Cuadra, S. F. Zappe, J. S. Minden, and J. KovaKovačević, "Towards an image analysis toolbox for high-throughput drosophila embryo RNAi screens," in Proc. IEEE Int. Symp. Biomed. Imaging, Arlington, VA, Apr. 2007, pp. 288-291.

[8] H. S. Malvar, Signal Processing With Lapped Transforms. Norwood, MA: Artech House, 1992.

[9] A. Chebira and J. Kovačević, "Lapped tight frame transforms," in Proc. IEEE Int. Conf. Acoust., Speech, Signal Process. (ICASSP), Honolulu, HI, Apr. 2007, vol. III, pp. 857-860.

[10] J. Gauthier, L. Duval, and J.-C. Pesquet, "Low redundancy oversampled lapped transforms and application to 3-D seismic data filtering," in Proc. IEEE Int. Conf. Acoust., Speech, Signal Process. (ICASSP), Toulouse, France, May 2006, vol. II, pp. 821-824.

[11] R. W. Young and N. G. Kingsbury, "Frequency-domain motion estimation using a complex lapped transform," IEEE Trans. Image Process., vol. 2, no. 1, pp. 2-17, Jan. 1993.

[12] J. Gauthier, L. Duval, and J.-C. Pesquet, "Oversampled inverse complex lapped transform optimization," in Proc. IEEE Int. Conf. Acoust., Speech, Signal Process., Apr. 2007, vol. I, pp. 549-552.

[13] J. Gauthier, L. Duval, and J.-C. Pesquet, "A non separable 2-D complex modulated lapped transform and its applications to seismic data filtering," in Proc. Eur. Signal Process. Conf., Sep. 2005.
[14] Z. Cvetković and M. Vetterli, "Tight Weyl-Heisenberg frames in $\ell^{2}(\mathbb{Z})$," IEEE Trans. Signal Process., vol. 46, no. 5, pp. 1256-1259, May 1998 .

[15] H. S. Malvar, "A modulated complex lapped transform and its applications to audio processing," in Proc. IEEE Int. Conf. Acoust., Speech, Signal Process. (ICASSP), Phoenix, AZ, Mar. 1999, pp. 1421-1424.

[16] J. Princen, A. Johnson, and A. Bradley, "Subband transform coding using filter bank designs based on time domain aliasing cancellation," in Proc. IEEE Int. Conf. Acoust., Speech, Signal Process. (ICASSP), Dallas, TX, Apr. 1987, pp. 2161-2164.

[17] J. J. Benedetto and M. C. Fickus, "Finite normalized tight frames," $A d v$. Comp. Math. (Special Issue on Frames), vol. 18, pp. 357-385, 2003.

[18] N. I. Akhiezer and I. M. Glazman, Theory of Linear Operators in Hilbert Space. Mineola, NY: Dover, 1993.

[19] D. Han and D. R. Larson, "Frames, bases and group representations," Memoirs Amer. Math. Soc., vol. 147, no. 697, pp. 1-94, 2000.

[20] M. Püschel and J. Kovačević, "Real, tight frames with maximal robustness to erasures," in Proc. Data Compr. Conf., Snowbird, UT, Mar. 2005, pp. 63-72.

[21] V. K. Goyal, J. Kovačević, and J. A. Kelner, "Quantized frame expansions with erasures," J. Appl. Comput. Harmon. Anal., vol. 10, no. 3, pp. 203-233, May 2001.

[22] R. N. Bracewell, "Discrete Hartley transform," J. Opt. Soc. Amer., vol. 73, no. 12, pp. 1832-1835, 1983.

[23] T. Kailath, Linear Systems. Englewood Cliffs, NJ: Prentice-Hall, 1980.

[24] S. Egner and M. Püschel, "Automatic generation of fast discrete signal transforms," IEEE Trans. Signal Process., vol. 49, no. 9, pp. 1992-2002, 2001.

[25] M. Püschel and J. M. F. Moura, "The algebraic approach to the discrete cosine and sine transforms and their fast algorithms," SIAM J. Comput., vol. 32, no. 5, pp. 1280-1316, 2003.

[26] T. F. Coleman and Y. Li, "An interior, trust region approach for nonlinear minimization subject to bounds," SIAM J. Optim., vol. 6, pp. 418-445, 1996.

[27] R. K. Brayton, S. W. Director, G. D. Hachtel, and L. Vidigal, "A new algorithm for statistical circuit design based on quasi-Newton methods and function splitting," IEEE Trans. Circuits Syst., vol. 26, pp. 784-794, 1979.

[28] K. Fan and A. J. Hoffman, "Some metric inequalities in the space of matrices," Trans. Amer. Math. Soc., vol. 6, no. 1, pp. 111-116, Feb. 1955.

[29] R. G. Douglas, "On majorization, factorization, and range inclusion of operators on Hilbert space," Trans. Amer. Math. Soc., vol. 17, pp. 413-415, 1966.

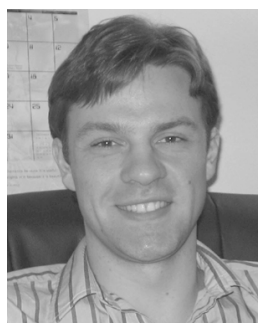

Aliaksei Sandryhaila (S'06) received the B.S. degree in computer science from Drexel University, Philadelphia, PA, in 2005 and the M.S. degree in electrical and computer engineering from Carnegie Mellon University (CMU) Pittsburgh, PA, in 2009. $\mathrm{He}$ is currently working towards the Ph.D. degree in electrical and computer engineering at CMU.

His research interests include signal processing, multiresolution analysis and filterbank design, applied mathematics, and fast algorithm design.

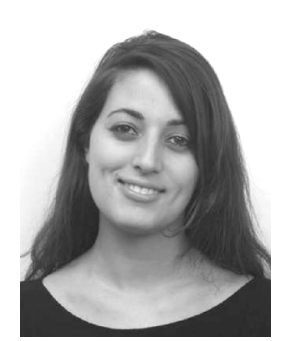

Amina Chebira (S'04-M'09) received the M.S. degree in communication systems from the Ecole Polytechnique Fédérale de Lausanne (EPFL), Switzerland, in 2003 and the Ph.D. degree from the Biomedical Engineering Department at Carnegie Mellon University, Pittsburgh, PA, in 2008.

From March to September 2003, she was a research intern at Philips National Research Laboratories, Eindhoven, The Netherlands. From 2003 to 2004, she was a Scientific Assistant at the Audiovisual Communications Laboratory at EPFL, where she is currently a Postdoctoral Researcher. Her research interests include frame theory, biomedical signal processing, and pattern recognition.

Dr. Chebira is an Associate Member of the Bio Imaging and Signal Processing Technical Committee of the IEEE Signal Processing Society. 


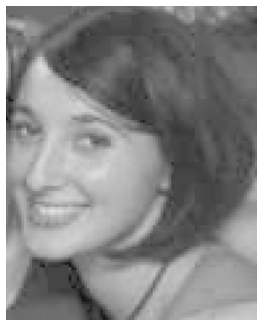

Christina Milo received the B.S. degree in electrical and computer engineering and the M.S. degree from Carnegie Mellon University (CMU), Pittsburgh, PA, in 2008 and 2009, respectively.

Her research interests include speech processing, image processing and machine learning.

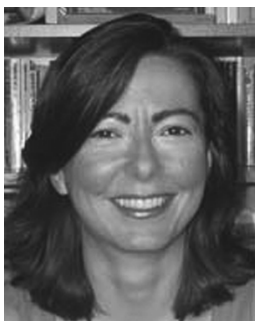

Jelena Kovačević (S'88-M'91-SM'96-F'02) received the Dipl.Electr.Eng. degree from the Electrical Engineering Department, University of Belgrade, Yugoslavia, in 1986 and the M.S. and Ph.D. degrees from Columbia University, New York, in 1988 and 1991, respectively.

She is a Professor of Biomedical Engineering and Electrical and Computer Engineering and the Director of the Center for Bioimage Informatics, Carnegie Mellon University, Pittsburgh, PA. Her research interests include bioimaging as well as multiresolution techniques such as wavelets and frames. From 1991 to 2002, she was with Bell Labs, Murray Hill, NJ. She was a co-founder and Technical VP of xWaveforms, based in New York City. She was also an Adjunct Professor at Columbia University. In 2003, she joined Carnegie Mellon University. She is a coauthor (with M. Vetterli) of the book Wavelets and Subband Coding (Prentice-Hall, 1995).

Dr. Kovačević coauthored a top-10 cited paper in the Journal of Applied and Computational Harmonic Analysis, and the paper for which A. Mojsilovic received the Young Author Best Paper Award. Her paper on multidimensional filter banks and wavelets (with M. Vetterli) was selected as one of the Fundamental Papers in Wavelet Theory. She received the Belgrade October Prize in 1986 and the E. I. Jury Award at Columbia University in 1991. She was the Editor-in-Chief of the IEEE TRANSACTIONS ON IMAGE PROCESSING. She served as an Associate Editor of the IEEE TRANSACTIONS ON SIGNAL PROCESSING, as a Guest Co-Editor (with I. Daubechies) of the Special Issue on Wavelets of the Proceedings of the IEEE, Guest Co-Editor (with M. Vetterli) of the Special Issue on Transform Coding of the IEEE Signal Processing Magazine, and Guest Co-Editor (with R. F. Murphy) of the Special Issue on Molecular and Cellular
Bioimaging of the IEEE Signal Processing Magazine. She has been on the Editorial Boards of the Foundations and Trends in Signal Processing, the SIAM book series on Computational Science and Engineering, the Journal of Applied and Computational Harmonic Analysis, the Journal of Fourier Analysis and Applications, and the IEEE Signal Processing Magazine. She is a regular member of the NIH Microscopic Imaging Study Section. From 2000 to 2002, she served as a Member-at-Large of the IEEE Signal Processing Society Board of Governors. She is the Chair of the Bio Imaging and Signal Processing Technical Committee. She was the General Chair of ISBI 2006, General Co-Chair (with V. Goyal) of the DIMACS Workshop on Source Coding and Harmonic Analysis, and General Co-Chair (with J. Allebach) of the Ninth IMDSP Workshop. She was a plenary/keynote speaker at the 20 Years of Wavelets 2009, European Women in Mathematics 2009, MIAABWorkshop 2007, Statistical Signal Processing Workshop 2007, Wavelet Workshop 2006, NORSIG 2006, ICIAR 2005, Fields Workshop 2005, DCC 1998, as well as SPIE 1998.

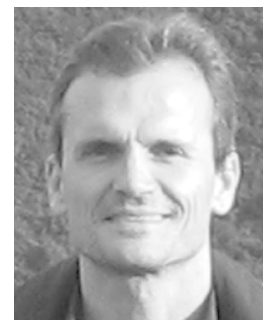

Markus Püschel (M'99-SM'05) received the Diploma (M.Sc.) degree in mathematics and the Ph.D. degree in computer science, in 1995 and 1998 , respectively, both from the University of Karlsruhe, Germany.

From 1998 to 1999 , he was a Postdoctoral Researcher at Mathematics and Computer Science, Drexel University. Since 2000 he has been with Carnegie Mellon University, Pittsburgh, PA, where he is currently a Professor of Electrical and Computer Engineering. His research interests include fast computing, algorithms, applied mathematics, and signal processing theory/software/hardware.

Dr. Püschel was an Associate Editor for the IEEE TRANSACTIONS ON SIGNAL PROCESSING and the IEEE SignAl Processing LeTters. He was a Guest Editor of the Proceedings of the IEEE and the Journal of Symbolic Computation and served on various program committees of conferences in computing, compilers, and programming languages. He is a recipient (with J. Moura) of the Outstanding Research Award of the College of Engineering at Carnegie Mellon and the Eta Kappa Nu Award for Outstanding Teaching. He also holds the title of Privatdozent (by habilitation) at the University of Technology, Vienna, Austria. He was a keynote/plenary speaker at the ACM ISSAC 2009, ACM GPCE 2007, iWAPT workshop 2007, and POHLL/IPDPS workshop 2007. 